\title{
A CORRECÃO DO DRIFT INSTRUMENTAL EM ICP-AES COM ESPECTRÔMETRO SEQÜENCIAL E A ANÁLISE DE ELEMENTOS MAIORES, MENORES E TRAÇOS EM ROCHAS
}

\author{
V.A.Janasi, S.Andrade, H.H.G.J.Ulbrich
}

PALAVRAS-CHAVE: espectrometria de plasma ICP-AES, geoquímica analítica, materiais de referência em rochas com valores certificados e correção de drifi.

JANASI, V.A. et al. (1995) A correção do driff instrumental em ICP-AES com espectrômetro seqüencial e a análise de elementos maiores, menores e traços em rochas. Bol. IG-USP, Sér. Cient., 26:45-58.

\section{RESUMO}

Apresentam-se resultados de análises quimicas de elementos maiores e traços $\mathrm{em}$ materiais de referência de rochas, obtidos durante o desenvolvimento de rotina analítica no Laboratório de Quimica e ICP-AES do Departamento de Mineralogia e Petrologia do Instituto de Geociéncias da USP. As análises, realizadas em equipamento ARL-3410 dotado de espectrómetro seqũencial, foram efetuadas em soluçōes obtidas por fusão alcalina, com misturas de $0,75 \mathrm{~g}$ de tetra e metaborato de lítio e de $0,25 \mathrm{~g}$ de pó de rocha, e diluiçăo final dos analitos de 1:1000 (metodologia do Imperial College, Londres). Para minimizar os efeitos de driff, provenientes especialmente de variaçőes nas condiçōes de introduçăo da amostra no plasma, adotam-se procedimentos rigidos de controle e correção off-line da flutuação do sinal. Os resultados apresentam boa repetibilidade e precisẫo a longo intervalo, refletindo a eficiéncia usualmente alcancada pelo procedimento adotado. Em vista da dificuldade de corrigir o drift de pequeno intervalo em plasmas dotados de espectrómetro seqūencial, os fechamentos das análises são variáveis entre 98 e $101 \%$, mas as relaçōes entre os elementos maiores se mantèm constantes, garantindo a qualidade dos resultados. A exatidão é satisfatória para todo o conjunto de elementos por ora estudado (além dos maiores e menores, ainda $\mathrm{Zn}, \mathrm{Ni}, \mathrm{Cr}, \mathrm{V}, \mathrm{Zr}, \mathrm{Sc}, \mathrm{Y}, \mathrm{La}, \mathrm{Sr}$ e Ba), mesmo para aqueles de determinaçầo mais dificil no ICP-AES, como K, P e La.

\section{ABSTRACT}

Analytical results of major, minor and trace elements are presented, which were obtained on international rock reference materials during the development of work routines in the ICPAES Laboratory, Mineralogy and Petrology Department, Instituto de Geociências, São Paulo University. Analyses were performed on an ARL-3410 model with sequential spectrometer using solutions obtained by alkaline fusion of $0.25 \mathrm{~g}$ of rock powder with $0.75 \mathrm{~g}$ of lithium tetra and metaborate (eutectic mixture), with a 1:1000 sample dilution (method used at the Imperial College, London). In order to minimize the effects of intensity drift, caused mostly by variations in the uptake conditions of the solution, strict procedures of drift control and off-line correction were adopted. The results show good repeatability and maintain long-term precision, demonstrating that reliable analyses can be obtained by careful correction procedures. However, as it is not possible to control short-term drift in sequential spectrometers, most of the analyses add up to between 98 and $101 \%$; key major element ratios are nevertheless maintained, ensuring the quality of the analysis. The accuracy is reliable for the analyzed elements, which, besides

Departamento de Mineralogia e Petrologia, Instituto de Geociências/USP, Sāo Paulo, Brasil. 
major and minor elements, also include $\mathrm{Zn}, \mathrm{Ni}, \mathrm{Cr}, \mathrm{V}, \mathrm{Zr}, \mathrm{Sc}, \mathrm{Y}, \mathrm{La}, \mathrm{Ba}$ and $\mathrm{Sr}$, and even for those notoriously difficult to analyze by ICP-AES, such as $\mathrm{K}, \mathrm{P}$ and $\mathrm{La}$.

\section{INTRODUÇÃO}

A espectrometria de emissão atômica com fonte de plasma induzido (ICP-AES) encontra atualmente larga aplicação na análise química de materiais geológicos, dada a sua reconhecida capacidade de produzir dados com rapidez e qualidade para uma ampla gama de elementos químicos. A técnica é amplamente reconhecida como excelente para a determinação de vários elementos traços, e em particular para a dos elementos terras-raras (ETR), caso em que substitui com vantagens as determinações via diluição isotópica ou análise instrumental com ativação neutrônica (INAA), mais demoradas e certamente mais caras (e.g., Potts, 1987). A determinação rotineira e precisa dos ETR permitiu, de fato, a sua utilização como valiosos indicadores petrogenéticos na literatura internacional e também nos trabalhos de pesquisa realizados em território brasileiro, principalmente após a implantação pioneira, por parte da Geosol Ltda., da rotina analítica para esses elementos (cf. Dutra, 1984; Figueiredo, 1985). A análise de elementos maiores, menores e vários outros traços por ICP-AES é também amplamente empregada, constando atualmente dos pacotes analíticos oferecidos pela maioria dos laboratórios geoquímicos a nivel mundial.

Para alcançar a elevada exatidão requerida na análise de elementos maiores (idealmente, melhor que $1 \%$ ), contu- do, fazem-se necessários cuidadosos procedimentos de controle da flutuação (drift) do sinal, por exemplo com a utilização de padrões internos (e.g., Walsh, 1992). Os resultados assim obtidos mostram, em geral, exatidão e precisão* iguais ou em alguns casos superiores aos determinados por técnicas tradicionais. Um exemplo extensamente documentado aparece na cuidadosa análise realizada por Gladney et al. (1990) para chegar aos valores recomendados (mais de 70 elementos) para o material de referência (Potts et al., 1992) Basalto BCR-1, do Serviço Geológico de EUA; nesse trabalho, a grande maioria dos valores obtidos rotineiramente por técnicas ICP-AES não mostram diferenças significativas com os determinados por meio de metodologias geralmente consideradas mais precisas (FRX, INAA, etc.). Excetuam-se dessa lista, claro está, os elementos para os quais a metodologia é insensivel ou não recomendada (tais como $\mathrm{Cl}, \mathrm{B}$ e $\mathrm{Rb}$ ) ou para cuja determinaçăo deve-se partir para preparativos técnicos adicionais $(\mathrm{Ta}, \mathrm{Nb}, \mathrm{W}$, $\mathrm{Pb}, \mathrm{Hg}, \mathrm{Sn}, \mathrm{Sb}, \mathrm{As}, \mathrm{Bi}$, etc.), via geração de hidretos e outros.

$O$ equipamento utilizado no Laboratório de Química/ICP do DMP-IG, da USP, é um modelo ARL-3410 seqüencial, utilizando nebulizador convencional, tipo Meinhard (cf. condições analiticas na Tabela 1). $\mathrm{O}$ desenvolvimento da rotina analítica para elementos maio-

\footnotetext{
*Exatidăo (accuracy) relata a concordância entre uma medida de concentração obtida $e$ valor verdadeiro da amostra de referència. Precisăo (precision): repetibilidade mede a variaçăo (expressa em termos de desvio-padrão) obtida em análises de uma mesma amostra no mesmo instrumento, e é parâmetro importante de medida da eficiência do controle do drift instrumental; precisão: reprodutibilidade refere-se à variação de valores (também expressa $\mathrm{em}$ termos de desvio-padrão) observada em diferentes determinaçōes de uma mesma amostra em diferentes equipamentos (IUPAC, 1978).
} 
Tabela 1- Condições operacionais e parâmetros instrumentais.

-Potência de saída: $650 \mathrm{~W}$

-Frequência: $60 \mathrm{~Hz}$

-Fluxo do gás plasma: $0,9 \mathrm{~L} / \mathrm{min}$

-Fluxo do gás transportador ("carrier"): $0,8 \mathrm{~L} / \mathrm{min}$

-Fluxo do gás refrigerador ("coolant"): $7,5 \mathrm{~L} / \mathrm{min}$

-Taxa de ascenção da solução ("uptake rate"): $2,4 \mathrm{~mL} / \mathrm{min}$

-Altura da leitura óptica: $6 \mathrm{~mm}$ acima da espira

-Nebulizador: tipo Meinhard (convencional)

res e menores no equipamento respondeu a uma necessidade imediata do Laboratório, e precisou resolver uma série de problemas técnicos, experiência que motiva o presente trabalho. De fato, praticamente todos os laboratórios onde se obtêm análises de elementos maiores por ICP-AES utilizam um equipamento com espectrômetro simultâneo (e.g. Cantagrel \& Pin, 1994). A determinação seqüencial dos elementos inviabiliza o controle das flutuações de pequeno intervalo (short-term drift) através de padrōes internos, além de acentuar a necessidade de se adotar cuidadosos procedimentos de controle para aquelas de longo intervalo (long-term drift), por aumentar significativamente o tempo de análise.

\section{PROCEDIMENTO DE ABERTURA DE AMOSTRAS}

A abertura das amostras é feita por fusão alcalina do pó da rocha com mistura de meta- e tetraborato de lítio. $\mathrm{O}$ método, além da relativa rapidez do processo de preparaçăo de soluçōes, oferece algumas vantagens importantes sobre o ataque ácido na análise de elementos maiores, entre elas a manutenção do Si em solução e a atenuação de possiveis efeitos de matriz, em vista do amplo predomínio do fundente na solução final. Adicionalmente, a fusão alcalina garante a dissolução de minerais refratários como zircão, cromita, berilo, monazita, e outros, permitindo portanto a quantificação de elementos traços como $\mathrm{Zr}$, $\mathrm{Cr}$ e $\mathrm{Be}$, sempre que presentes em teores adequados (e.g., Jarvis \& Jarvis, 1992).

O método adotado segue, com pequenas modificações, os procedimentos desenvolvidos na Analytical Facility do Departamento de Ciências Geológicas do Imperial College de Londres, gentilmente fornecidos por Peter J. Watkins (com. pessoal, 1994).

Cerca de $2,0 \mathrm{~g}$ de amostra com granulação abaixo de 200 mesh são pesados analiticamente em cadinhos de porcelana, e levados à estufa a $100^{\circ} \mathrm{C}$, por algumas horas (dependendo do tempo disponível do operador e dos procedimentos adotados em cada laboratório). Numa das rotinas, deixa-se o material na estufa por cerca de 12 horas (da noite para o dia seguinte), de onde, uma vez retirado, é levado para dessecador até que a temperatura se reequilibre com o ambiente (cerca de 60 minutos). Os cadinhos são repesados, e a diferença percentual de massa observada corresponde à perda de umidade $\left(\mathrm{H}_{2} \mathrm{O}^{-}\right)$.

A determinação de perda ao fogo pode ser feita, evidentemente, de várias maneiras para a grande maioria das rochas que mostrem baixos teores de água: simultaneamente à determinação anterior, pesando cerca de $2 \mathrm{~g} \mathrm{em} \mathrm{ca-}$ dinhos separados (alguns autores recomendam utilizar $4 \mathrm{~g}$ ), ou em seqüência. Neste último procedimento, é retirada uma aliquota de $0,25 \mathrm{~g}$, a ser utilizada para realizar a operaçã̃o de fusão. O pó restante, cerca de $1,75 \mathrm{~g}$, é depositado em cadinho previamente tarado e levado à mufla a $1000^{\circ} \mathrm{C}(1$ ou $2 \mathrm{~h})$, colocado em dessecador após a retirada da mufla até reequilibrio térmico com o meio, e 
pesado: a diferenca de massa corresponde, neste caso, diretamente à perda ao fogo (no primeiro caso, o da operação simultânea, deve ser efetuada uma correção por perda de umidade). A perda ao fogo de materiais ricos em água, carbonatos, etc., deve ser feita por aquecimento lento em mufla, para impedir perda explosiva de material durante a desidratação, decarbonatação, etc.

A fusão alcalina emprega 0,2500 $\pm 0,0001 \mathrm{~g}$ da amostra (pesados em outro cadinho de porcelana), aos quais se adiciona $0,7500 \pm 0,0003 \mathrm{~g}$ de fundente, constituído por mistura eutética de metaborato de lítio e tetraborato de lítio (na proporçăo 4:1, com ponto eutético a $832^{\circ} \mathrm{C}$; Sulcek \& Povondra, 1989); vários outros fundentes a base de boratos, com proporçōes diferentes às indicadas, são também recomendadas na literatura e podem ser obtidas comercialmente (tipo "Spectromelt", etc.; ver obra cit.). Amostra e fundente são cuidadosamente homogeneizados com bastôes de vidro e transferidos para cadinhos de grafite. A fusăo, nesses cadinhos, é feita em forno pré-aquecido a $1000^{\circ} \mathrm{C}$, por 20 minutos. A massa fundida, movimentada no cadinho por meio de rotaçōes para garantir a formação de pérola única com a coalescência de outras pérolas menores, é então diretamente despejada em béquer plástico de $400 \mathrm{~mL}$ contendo solução ácida $(150 \mathrm{~mL}$ de água destilada e 25 $\mathrm{mL}$ de $\mathrm{HNO}_{3} 2 \mathrm{~N}$ ). A pérola converte-se assim em vidro, e deve ser imediatamante dissolvida, com o auxilio de bastőes magnéticos, em placa agitadora; os vidros mais refratários das rochas mais comuns (basaltos, granitóides, dioritos, etc.) não demoram mais que 30 minutos até dissolução completa. Em alguns casos, são observadas pequenas pérolas residuais retidas nos cadinhos, que devem ser cuidadosamente transferidas para o béquer com a soluçâo correspondente, por meio de raspagem com espátula de metal.
A seguir, a solução é transferida para balōes volumétricos de $250 \mathrm{~mL}$; o volume é completado com a água de lavagem dos béquers e água destilada. $\mathrm{A}$ solução é finalmente filtrada em filtro de papel rápido (tipo Whatmann $n^{\circ} 41$, ou similar, para retirar partículas de grafite) quando da transferência para frascos de polipropileno de $250 \mathrm{ml}$, onde é estocada até a análise. A observaçāo corriqueira parece mostrar que essa estocagem pode se estender por vários meses, sem que mudem as características químicas da solução e, conseqüentemente, sejam deturpadas as relaçōes analíticas obtidas em determinaçōes espaçadas no tempo. As soluçōes resultantes têm uma diluição $1: 1000$ dos analitos, sendo compostas de $1000 \mathrm{ppm}$ de amostra e $3000 \mathrm{ppm}$ de fundente em soluçẫo $\mathrm{HNO}_{3} \quad 0,2 \mathrm{~N}$. Uma aliquota de cerca de $50 \mathrm{~mL}$ da solução final é separada para análise de elementos traços. A esta alíquota são adicionadas 2 gotas (ca. $0,1 \mathrm{~mL}$ ) de HF $48 \%$, para impedir a possível precipitação de alguns elementos traços tais como o $\mathrm{Zr}$, que são mantidos $\mathrm{cm}$ solução pela formação de complexos com o F.

As soluçōes obtidas em amostras de rochas no intervalo composicional granito-basalto são normalmente limpidas, e não deixam residuos de minerais insolúveis. Por vezes, pode haver precipitação de gel silicoso, em especial quando o procedimento de dissoluçăo descrito acima não é iniciado imediatamente após o resfriamento do vidro; nesse caso, a amostra tem que ser preparada novamente. Servirá como branco uma solução adicional, preparada em paralelo com cada conjunto de amostras, apenas por fusão e solubilizaçẫo de $0,7500 \mathrm{~g}$ de mistura de boratos. Os materiais de referência são preparados independentemente, por meio do procedimento indicado, para utilização como definidores de curvas de calibração ou mesmo para serem inseridos na rotina 
analítica como soluçð̃es-problema para controle da qualidade do trabalho.

Tabela 2- Linhas utilizadas, posições de leitura de background $\left(\mathrm{L}_{B g}\right)$ e limites de detecção $\left(\mathrm{L}_{\mathrm{d}}\right)$ e de quantificação $\left(\mathrm{L}_{\mathrm{q}}\right)$ para elementos maiores.

\begin{tabular}{|c|c|c|c|c|}
\hline Analito & $\begin{array}{l}\text { Compr. de } \\
\text { onda }(\mathrm{nm})\end{array}$ & $\begin{array}{l}\mathrm{L}_{B_{g}} \\
(\mathrm{~nm})\end{array}$ & $\begin{array}{c}L_{d(k-G)} \\
(\%)\end{array}$ & $\begin{array}{c}\mathrm{L}_{\mathrm{q}(0-10)} \\
(\%)\end{array}$ \\
\hline $\mathrm{Fe}_{2} \mathrm{O}_{3}$ & 259,940 (II) & & 0,002 & 0,004 \\
\hline $\mathrm{MgO}$ & 279.079 (II) & $+0,037$ & 0,016 & 0,020 \\
\hline $\mathrm{SiO}_{2}$ & $288,158(\mathrm{I})$ & & 0,06 & 0,10 \\
\hline $\mathrm{Al}_{2} \mathrm{O}_{3}$ & 308,215 (I) & & 0,020 & 0,040 \\
\hline $\mathrm{CaO}$ & 317,933(II) & & 0,004 & 0,005 \\
\hline $\mathrm{Na}_{2} \mathrm{O}$ & $588,995(\mathrm{I})$ & & 0,018 & 0,030 \\
\hline $\mathrm{K}_{2} \mathrm{O}$ & $766,491(\mathrm{I})$ & & 0,08 & 0,15 \\
\hline
\end{tabular}

(I): linha atômica neutra; (II): linha atômica monoiôica.

\section{ESTRATÉGIA ANALÍTICA ADO- TADA}

\section{Calibração}

As análises são feitas em duas corridas separadas. $\mathrm{Na}$ primeira, são analisados os sete elementos maiores ( $\mathrm{Si}$, $\mathrm{Al}, \mathrm{Fe}, \mathrm{Mg}, \mathrm{Ca}, \mathrm{Na}, \mathrm{K}$ ), além de $\mathrm{Li}(\mathrm{a}-$ penas como controle, por estar presente em excesso), e na segunda, nove elementos entre menores e traços $(\mathrm{Ti}, \mathrm{P}$, $\mathrm{Mn}, \mathrm{Ba}, \mathrm{Sr}, \mathrm{Zr}$, V, além de $\mathrm{La} \mathrm{e} \mathrm{Y}$ em rochas ácidas, ou $\mathrm{Cr}$ e $\mathrm{Ni}$ em rochas básicas). As linhas analíticas utilizadas são listadas nas Tabelas 2 e 3; correções de background são feitas apenas para alguns elementos $(\mathrm{Mg}, \mathrm{P}, \mathrm{Zr}, \mathrm{V}, \mathrm{La}, \mathrm{Y}$, Cr e Ni; Tabela 4).

As curvas de calibração são obtidas a partir de materiais de referência certificados (IUPAC, 1993 e Govindaraju, 1995), principalmente os que são oferecidos pelo GIT-IWG, Groupe International de Travail-International Working Group (Govindaraju, 1994). As curvas para elementos maiores são tipicamente obtidas a partir de 4 materiais de referência certificados mais o
Tabela 3- Linhas utilizadas, posições de leitura de background $\left(\mathrm{L}_{B g}\right)$ e limites de decisão $\left(\mathrm{L}_{c}\right)$ e de detecçào $\left(\mathrm{L}_{\mathrm{d}}\right)$ e de quantificação $\left(\mathrm{L}_{\mathrm{q}}\right)$ para elementos menores e traços.

\begin{tabular}{|c|c|c|c|c|}
\hline Analito & $\begin{array}{ll}\text { Compr.de } & \mathbf{L}_{B g} \\
\text { onda }(\mathrm{nm}) & (\mathrm{nm})\end{array}$ & $\begin{array}{l}I_{c(p-s)} \\
(\mathrm{ppm})\end{array}$ & $\begin{array}{l}\mathrm{L}_{d(n-6)} \\
(\mathrm{ppm})\end{array}$ & $\begin{array}{l}\mathrm{L}_{\mathrm{q}(\mathrm{k}-10)} \\
(\mathrm{ppm})\end{array}$ \\
\hline $\mathrm{P}_{2} \mathrm{O}_{5}$ & $213,618(I)+0,020$ & 135 & 270 & 450 \\
\hline $\mathrm{Ni}$ & 231,604 (II) $+0,032$ & 10 & 20 & 35 \\
\hline $\mathrm{MnO}$ & 257,610 (II) & 2 & 4 & 5 \\
\hline $\mathrm{Cr}$ & 267,716 (II) $+0,032$ & 8 & 16 & 25 \\
\hline V & 290,880 (II) $-0,024$ & 6 & 12 & 20 \\
\hline $\mathrm{TiO}_{2}$ & 337,280 (II) & 9 & 18 & 30 \\
\hline $\mathrm{Zr}$ & 339,198 (II) $-0,029$ & 5 & 10 & 15 \\
\hline Sc & 361,359 (II) $+0,024$ & 1 & 2 & 3 \\
\hline Y & 371,030 (II) $+0,035$ & 2 & 4 & 7 \\
\hline $\mathrm{La}$ & 398,852 (II) $-0,029$ & 15 & 30 & 50 \\
\hline $\mathrm{Sr}$ & 407,771 (II) & 0,3 & 0.6 & 1 \\
\hline $\mathrm{Ba}$ & 455,403 (II) & 1 & 2 & 3 \\
\hline
\end{tabular}

(I), (II): ver observaçăo na Tabela 2.

Tabela 4 - Principais interferências identificadas a partir de soluçőes puras nas linhas analíticas utilizadas

\begin{tabular}{lccccc} 
Elementos & \multicolumn{5}{c}{ Elementos Interferentes } \\
Interferidos & $\mathrm{Fe}_{2} \mathrm{O}_{3}$ & $\mathrm{Al}_{2} \mathrm{O}_{3}$ & $\mathrm{CaO}$ & $\mathrm{MgO}$ & $\mathrm{TiO}_{2}$ \\
$\mathrm{Zn}(\mathrm{ppm})$ & 20,2 & & & \multicolumn{1}{c}{19} \\
$\mathrm{Cr}(\mathrm{ppm})$ & & & & & 5,2 \\
$\mathrm{~V}(\mathrm{ppm})$ & 5 & & & & \\
$\mathrm{La}(\mathrm{ppm})$ & & & 8,4 & & \\
$\mathrm{Ni}(\mathrm{ppm})$ & 5,5 & & & & \\
$\mathrm{P}_{2} \mathrm{O}_{5}(\%)$ & & 0,004 & & & 0,006 \\
$\mathrm{~K}_{2} \mathrm{O}(\%)$ & & & 0,19 & 0,14 &
\end{tabular}

Teores dos elementos interferidos calculados em curvas de calibração obtidas a partir de valores estipulados dos interferentes (10\% para todos os óxidos, exceto $\mathrm{TiO}_{2}, 5 \%$ ). As linhas utilizadas para os demais elementos náo apresentam interferências significativas.

branco. Os materiais mais frequientemente empregados são os granitos $\mathrm{GH}$ e $\mathrm{GA}$, o diorito DR-N e o basalto BE-N, que cobrem satisfatoriamente $O$ espectro composicional das amostras analisadas, exceto para $\circ \mathrm{Na}$, que tem seu ponto 
mais alto definido pelo granito $\mathrm{AC}-\mathrm{E}$. Para os elementos traços, emprega-se gama maior destes materiais de referência, e normalmente menor número de pontos na curva ( 2 ou 3 mais o branco). Em alguns casos, os pontos de alta concentração para alguns elementos traços sâo obtidos por adição de concentração conhecida do elemento de interesse em soluçōes de materiais certificados nos quais este está presente em teor baixo.

Em função da resposta linear da metodologia, que constitui uma das suas maiores vantagens (ver Thompson \& Walsh, 1989, e literatura ali citada), as curvas que relacionam concentração $e$ intensidade são sempre equaçōes de primeiro grau, obtidas como:

\section{Concentraçảo $(\%$ ou ppm $)=a+b x$ Intensidade (em kilocontageris)}

com coeficientes de correlação que devem ser superiores a 0,9997 ; de fato, estes coeficientes constituem critério de avaliação da qualidade da curva de calibração e, por conseguinte, do procedimento de preparação dos padrões e do adequado funcionamento do próprio equipamento. Quando os teores esperados nas amostras-problema são baixos, trabalha-se com curvas nas quais:

\section{Concentraçăo (\% ou ppm) $=\mathrm{a}+\mathrm{bxl} /$ Intensidade}

melhorando-se com isto a exatidão para valores próximos de zero; devem ser esperados, também neste caso, altos coeficientes de correlação.

O software do equipamento permite também o traçado de polinômios de segundo ou terceiro graus. Entretanto, a sua utilização, em lugar da equação de primeiro grau, deve ser desencorajada, exceto para aqueles elementos que, em alta concentração, mostrariam respostas nâo lineares (e.g., $\mathrm{Ca}$ em rochas ricas nesse elemento e principalmente
$\mathrm{Mg}$ em rochas ultramáficas com teores elevados do elemento; cf., por exemplo, Thompson \& Walsh, 1989). Com efeito, um espalhamento excessivo de pontos na curva de calibração para determinado elemento representa, em geral, erros vários (e.g., determinação deficiente do pico do elemento em questão; soluções inadequadamente preparadas de padrōes; possivelmente até imprecisões nos valores recomendados do elemento, difundidas na literatura sobre materiais nâo certificados; etc.), erros estes que evidentemente não serão corrigidos artificialmente por meio de uma suposta melhor representação através desses polinômios.

\section{Correção do sinal}

Um dos principais problemas enfrentados em análises por espectrometria de plasma é a existência de significativo drift do sinal. Tais problemas são particularmente importantes quando o sinal é detectado em espectrômetros scqüenciais, que tornam desaconselhável a utilização de padrōes internos, estratégia crescentemente reconhecida como importante na análise de elementos maiores (e.g., Walsh \& Howie, 1980; Walsh, 1992).

O drift do sinal pode resultar de uma série de fatores, dos quais os de maior incidência são: (1) mudanças na transferência de energia do plasma para a amostra (em geral vinculadas a instabilidades eletrônicas), (2) variaçōes na eficiência de produção e transporte da amostra e (3) degradação da medição das intensidades da linha (em geral ligada a drift térmico do sistema óptico) (e.g., Carré et al., 1992; Walsh, 1992). Existem na literatura poucos trabalhos dedicados ao diagnóstico das causas especificas do drift em ICP-AES, mas em casos favoráveis ele pode ser feito de forma relativamente simples, pela análise do comportamento de linhas mais e menos sensiveis a variaçōes nos parâ- 
metros citados (e.g., Carré et al., 1992). Em termos gerais, contudo, existe consenso de que o fator (2) acima é o principal responsável pelo drift na maior parte dos casos (Walsh, 1992). As causas da maior ou menor eficiência de nebulização podem ser várias, citando-se entre elas variaçōes no fluxo do gás transportador ou na taxa de ascenção da soluçâo, bloqueios parciais do nebulizador (por deposiçāo de sais ou de minúsculas partículas de grafite provenientes da solução analisada), e mudanças na temperatura da soluçăo e/ou da câmara de nebulização (spray chamber) (Carré et al., 1992). Vários esforços vêm sendo feitos no laboratório de ICP-AES do DMP-IG, da USP, para minimizar a magnitude do drifi, entre eles o controle da temperatura ambiente e o acompanhamento sistemático de instabilidades nos sistemas óptico e eletrônico do equipamento. Será instalada em breve uma câmara de nebulização termostatizada, que deverá produzir efeito significativo na redução do drift. $\mathrm{O}$ uso de bomba peristáltica para garantir uma taxa de ascensão mais constante foi desencorajado pelo significativo aumento do desvio-padrão relativo das medidas observado.

Como atualmente observados no laboratório, o tipo e a magnitude do drift são bastante variáveis de dia para dia, e mudam até para diferentes elementos ou mesmo para diferentes linhas do mesmo elemento; variações da ordem $\pm 3 \%$ em espaço de tempo de $2-3$ horas são comuns, e resultam em sensivel deterioração da qualidade analitica, particularmente dos elementos maiores, em que exatidões da ordem de $1 \%$ são requeridas.

Em vista disto, foram adotados procedimentos de controle off-line do drift, que envolvem a normalização dos sinais obtidos em relação à variação do sinal de uma amostra de controle. O procedimento finalmente adotado envol- ve uma sequiência na leitura das amostras-problema ( $\mathrm{AM})$, com intercalação de uma amostra de controle do drift (CD) a intervalos regulares (a cada 6 amostras, correspondendo a corrida total a cerca de 30 minutos, para análise de 8 elementos); como padrões para controle de qualidade, são também analisadas as soluções de dois materiais de referência certificados (PI1 e PI2). As soluções para análise apresentam-se então na seguinte ordem:

$$
\begin{gathered}
\mathrm{CD}(1)-\mathrm{AM}(1)-\mathrm{AM}(2)-\mathrm{AM}(3)-\mathrm{AM}(4)-\mathrm{AM}(5)- \\
\mathrm{PII} / / \mathrm{CD}(2)-\mathrm{AM}(6)-\mathrm{AM}(7)-\mathrm{AM}(8)-\mathrm{AM}(9)- \\
\mathrm{AM}(10)-\mathrm{BR} / / \mathrm{CD}(3)-\mathrm{AM}(11)-\mathrm{AM}(12)- \\
\mathrm{AM}(13)-\mathrm{AM}(14)-\mathrm{AM}(15)-\mathrm{AM}\left({ }^{* a}\right) / / \mathrm{CD}(4)- \\
\mathrm{AM}(16)-\mathrm{AM}(17)-\mathrm{AM}(18)-\mathrm{AM}(19)-\mathrm{AM}(20)-\mathrm{PI} 2 \\
/ / \mathrm{CD}(5)-\mathrm{AM}(21)-\mathrm{AM}(22)-\mathrm{AM}(23)-\mathrm{AM}(24)- \\
\operatorname{AM}(25)-\mathrm{AM}(* \mathrm{~b}) / / \mathrm{CD}(6) .
\end{gathered}
$$

e assim sucessivamente. As siglas CD $(n=1,2$, etc.) identificam a intensidade (em kilocontagens) da amostra de controle de drift na sequêencia 1,2 , etc., AM $(\mathrm{i}=1,2$, etc.) as intensidades das amostras-problema, BR a do branco e PIl e $\mathrm{PI} 2$ as dos materiais certificados; com asterisco, são indicadas as intensidades de duplicatas de algumas das amostras pesquisadas.

No procedimento adotado, a intensidade do sinal obtido para uma amostra AM, analisada entre duas leituras sucessivas de CD, é corrigida pela variação percentual do sinal entre essas duas leituras, ponderada pelo tempo decorrido entre a leitura de $C D(n)$ e a de AM. O valor assim corrigido é então referido à primeira contagem do controle do drift -a leitura $\mathrm{CD}(1)$ - o que se obtém pela multiplicação por $\mathrm{CD}(1) / \mathrm{CD}(\mathrm{n})$.

Este procedimento efetivamente melhora a repetibilidade dos resultados, mantendo o desvio-padrão relativo $\left(\mathrm{RSD}_{\mathrm{r}}\right.$ ) (IUPAC, 1990) tipicamente abaixo de $1 \%$ para a maioria dos elementos (cf. Tabelas 5 e 6). Entretanto, pode haver deterioração da exatidão dos 
Tabela 5 - Resultados das análises de elementos maiores em materiais de referência internacionais.

\begin{tabular}{|c|c|c|c|}
\hline Elemento & $\begin{array}{l}\text { Valor } \\
\text { Recom. }\end{array}$ & $\begin{array}{l}\text { Valor } \\
\text { Obtido }\end{array}$ & $s_{\mathrm{I}}$ \\
\hline $\mathrm{SiO}_{2}$ & 52.16 & 52.63 & 0.50 \\
\hline $\mathrm{Al}_{2} \mathrm{O}_{3}$ & 14.51 & 14.25 & 0.06 \\
\hline $\mathrm{Fe}_{2} \mathrm{O}_{3}$ & 9.10 & 9.05 & 0.03 \\
\hline $\mathrm{MgO}$ & 7.75 & 7.94 & 0.01 \\
\hline $\mathrm{CaO}$ & 9.23 & 9.23 & 0.08 \\
\hline $\mathrm{Na}_{2} \mathrm{O}$ & 2.74 & 2.75 & 0.03 \\
\hline $\mathrm{K}_{2} \mathrm{O}$ & 1.42 & 1.43 & 0.02 \\
\hline $\mathrm{A} / \mathrm{CNK}$ & 0.64 & 0.62 & \\
\hline mg & 62.80 & 63.50 & \\
\hline
\end{tabular}

\section{JA-1 $(\mathrm{n}=4)$}

Elemento

Valor Valor

Recom. Obtido

$\mathrm{SiO}_{2}$

$\mathrm{Al}_{2} \mathrm{O}_{3}$

$\mathrm{Fe}_{2} \mathrm{O}_{3}$

$\mathrm{MgO}$

$\mathrm{CaO}$

$\mathrm{Na}_{2} \mathrm{O}$

$\mathrm{K}_{2} \mathrm{O}$

A/CNK

mgH

\begin{tabular}{|c|c|c|c|c|}
\hline \multirow{3}{*}{ Elemento } & JG-1a & $(n=4)$ & & \\
\hline & Valor & Valor & & RSD \\
\hline & Recom. & Obtido & & \\
\hline $\mathrm{SiO}_{2}$ & 72.19 & 72.16 & 0.40 & \\
\hline $\mathrm{Al}_{2} \mathrm{O}_{3}$ & 14.22 & 14.00 & 0.16 & \\
\hline $\mathrm{Fe}_{2} \mathrm{O}_{3}$ & 2.05 & 2.02 & 0.02 & \\
\hline $\mathrm{MgO}$ & 0.69 & 0.71 & 0.01 & \\
\hline $\mathrm{CaO}$ & 2.13 & 2.17 & 0.02 & \\
\hline $\mathrm{Na}_{2} \mathrm{O}$ & 3.41 & 3.38 & 0.04 & \\
\hline $\mathrm{K}_{2} \mathrm{O}$ & 4.01 & 3.93 & 0.01 & \\
\hline $\mathrm{A} / \mathrm{CNK}$ & 1.03 & 1.02 & & \\
\hline mgd & 40.00 & 41.00 & & \\
\hline
\end{tabular}

\begin{tabular}{rrrr}
\multicolumn{4}{c}{ JA-2 $(\mathbf{n = 3})$} \\
Valor & Valor & s $_{\mathrm{r}}$ & RSD $_{\mathrm{r}}$ \\
Recom. & Obtido & & \\
56.18 & 56.76 & 0.56 & 0.99 \\
15.32 & 15.32 & 0.18 & 1.15 \\
6.14 & 6.33 & 0.16 & 2.54 \\
7.68 & 8.06 & 0.12 & 1.49 \\
6.48 & 6.18 & 0.17 & 2.81 \\
3.08 & 3.12 & 0.05 & 1.70 \\
1.80 & 1.78 & 0.04 & 2.27 \\
0.85 & 0.89 & & \\
71.20 & 71.60 & &
\end{tabular}

\section{JG-3 $(n=4)$}

RSD, Valor Recom. Valor s, RSD, Obtido

$\begin{array}{lll}67.96 & 0.11 & 0.17\end{array}$

$\begin{array}{lll}15.62 & 0.08 & 0.52\end{array}$

$\begin{array}{lll}3.76 & 0.05 \quad 1.40\end{array}$

$\begin{array}{lll}1.86 & 0.01 & 0.27\end{array}$

$\begin{array}{lll}3.73 & 0.01 & 0.15\end{array}$

$\begin{array}{lll}4.00 & 0.05 \quad 1.20\end{array}$

$\begin{array}{lll}2.60 & 0.10 \quad 3.78\end{array}$

2.63

0.95

0.96

49.50

48.70

\section{NIM-N $(\mathrm{n}=3)$}

Valor Valor $s_{\mathrm{r}}$ RSD.

Recom. Obtido

$\begin{array}{lllll}52.64 & 52.57 & 0.44 & 0.84\end{array}$

$\begin{array}{llll}16.50 & 16.33 & 0.04 & 0.27\end{array}$

$\begin{array}{lllll}8.91 & 9.09 & 0.07 & 0.81\end{array}$

$\begin{array}{lllll}7.50 & 7.58 & 0.01 & 0.08\end{array}$

$\begin{array}{llll}11.50 & 11.41 & 0.03 & 0.26\end{array}$

$\begin{array}{lllll}2.46 & 2.44 & 0.04 & 1.79\end{array}$

\begin{tabular}{l|lll}
0.25 & 0.20 & 0.02 & 10.24
\end{tabular}

$0.65 \quad 0.65$

$62.50 \quad 62.30$

Obs.: Valores recomendados săo os citados em Govindaraju (1994). $\mathbf{n}=$ número de observaçôes; $s_{r}$ e $\mathrm{RSD}_{1}$ săo desvio padrão e desvio padrão relativo na repetibilidade, respectivamente. $\mathrm{A} / \mathrm{CNK}=\mathrm{Al}_{2} \mathrm{O}_{3} /$ $\left(\mathrm{CaO}+\mathrm{Na}_{2} \mathrm{O}+\mathrm{K}_{2} \mathrm{O}\right)$ em proporçōes moleculares e mg\# $=100 \times \mathrm{Mg} /\left(\mathrm{Mg}+\mathrm{Fe}_{\mathrm{T}}\right) \mathrm{em}$ proporções atômicas.

resultados se ocorreu algum drift significativo do sinal entre a calibração e a leitura de $\mathrm{CD}(1)$. Como o tempo consumido na calibração e ajuste de curvas é relativamente grande (tipicamente, pouco mais de 20 minutos), é 
Tabela 6 - Resultados de análises dos elementos menores e traços em materiais de referência (JG-1a e JB-1a) e materiais de referência certificados (GS-N e GH).

\begin{tabular}{|c|c|c|c|c|}
\hline \multirow{2}{*}{ Elemento } & \multicolumn{2}{|c|}{ GS-N $(n=4)$} & \multirow[b]{2}{*}{$s_{t}$} & \multirow[b]{2}{*}{$\mathrm{RSD}_{\mathrm{r}}$} \\
\hline & $\begin{array}{l}\text { Valor } \\
\text { Recom. }\end{array}$ & $\begin{array}{l}\text { Valor } \\
\text { Obtido }\end{array}$ & & \\
\hline $\mathrm{TiO}_{2}$ & 0.68 & 0.68 & 0.01 & 1.48 \\
\hline $\mathrm{P}_{2} \mathrm{O}_{3}^{2}$ & 0.28 & 0.29 & 0.01 & 2.03 \\
\hline MnO & 0.06 & 0.06 & 0.00 & 0.00 \\
\hline $\mathrm{Ba}$ & 1400 & 1415 & 6 & 0.41 \\
\hline Sr & 570 & 585 & 8 & 1.29 \\
\hline $\mathrm{Zr}$ & 235 & 241 & 4 & 1.56 \\
\hline $\mathrm{La}$ & 75 & 87 & 10 & 11.53 \\
\hline $\mathrm{Y}$ & 16 & 12 & 6 & 47.32 \\
\hline V & 65 & 80 & 7 & 8.98 \\
\hline
\end{tabular}

\begin{tabular}{|c|c|c|c|}
\hline \multicolumn{4}{|c|}{ GH $(n=4)$} \\
\hline Valor & Valor & $s_{r}$ & $\mathrm{RSD}_{\mathrm{r}}$ \\
\hline Recom. & Obtido & & \\
\hline 0.08 & 0.07 & 0.00 & 6.90 \\
\hline 0.01 & 0.02 & 0.01 & 38.49 \\
\hline 0.05 & 0.05 & 0.00 & 0.00 \\
\hline 20 & 26 & 2 & 7.85 \\
\hline 10 & 16 & $i$ & 7.22 \\
\hline 150 & 172 & 2 & 1.01 \\
\hline 25 & 24 & 12 & 52.29 \\
\hline 75 & 78 & 2 & 2.18 \\
\hline 5 & $<20$ & & \\
\hline
\end{tabular}

\begin{tabular}{lrrrr} 
Elemento & $\begin{array}{r}\text { JG-1a } \\
\text { Valor } \\
\text { Recom. }\end{array}$ & $\begin{array}{c}(\mathbf{n = 4}) \\
\text { Valor } \\
\text { Obtido }\end{array}$ & \multicolumn{1}{c}{$\mathrm{s}_{\mathrm{r}}$} & \multicolumn{1}{c}{$\mathrm{RSD}_{\mathrm{r}}$} \\
$\mathrm{TiO}_{2}$ & 0.25 & 0.25 & 0.00 & 2.02 \\
$\mathrm{P}_{2} \mathrm{O}_{5}$ & 0.08 & 0.07 & 0.01 & 20.20 \\
$\mathrm{MnO}$ & 0.06 & 0.06 & 0.00 & 0.00 \\
$\mathrm{Ba}$ & 458 & 464 & 8 & 1.77 \\
$\mathrm{Sr}$ & 185 & 184 & 4 & 2.10 \\
$\mathrm{Zr}$ & 121 & 132 & 3 & 2.01 \\
$\mathrm{La}$ & 22 & 30 & 14 & 47.09 \\
$\mathrm{Y}$ & 32 & 30 & 1 & 3.85 \\
$\mathrm{~V}$ & 23 & 20 & &
\end{tabular}

preferivel referir os valores corrigidos não a $C D(1)$, mas ao valor de intensidade $\mathrm{CD}(0)$ que a amostra de controle, de composição conhecida, teria se perfeitamente ajustada às curvas de calibraç̃o; de fato, a experiência tem mostrado que a opção por normalizar os sinais de $\mathrm{CD}$ para o valor $\mathrm{CD}(0)$, calculado a partì da equação da curva de calibração, garante melhor exatidão.

A equação de correção adotada é da seguinte forma:

$$
\left.\mathrm{AM}_{(\mathrm{i})} *=\mathrm{AM}_{(\mathrm{i})} \times\left(\mathrm{CD}_{(0)}\right) \mathrm{CD}_{(\mathrm{n})}\right) \times\{1-\mathrm{A} \times \mathrm{B}\}
$$

onde:

$A=\left[\left(C D_{(n+1)}-C D_{(n)}\right) / C D_{(n+1)}\right]$

$B=\left[\left(\operatorname{LAM}_{(\mathrm{i})}-\operatorname{tCD}_{(\mathrm{n})}\right) /\left(\operatorname{tCD}_{(\mathrm{n}+1)}-\operatorname{tCD}_{(\mathrm{n})}\right)\right]$

Nas equações acima, as siglas $C D(n), C D(n+1)$ e $C D(0)$ representam as intensidades já indicadas, AM(i) e $\mathrm{AM}(\mathrm{i})^{*}$ são as intensidades da amostra i

\begin{tabular}{|c|c|c|c|}
\hline Valor & Valor & $s_{t}$ & $R_{S D}$ \\
\hline Recom. & Obtido & & \\
\hline 1.30 & 1.30 & 0.01 & 0.39 \\
\hline 0.26 & 0.26 & 0.01 & 2.26 \\
\hline 0.15 & 0.16 & 0.00 & 0.00 \\
\hline 497 & 514 & 1 & 0.22 \\
\hline 443 & 457 & 3 & 0.58 \\
\hline 146 & 137 & 4 & 2.75 \\
\hline 38 & 42 & 14 & 34.33 \\
\hline 24 & 23 & 1 & 4.44 \\
\hline 220 & 257 & 6 & 2.26 \\
\hline
\end{tabular}

sem correçāo e corrigida, e a letra $t$ identifica o tempo (minuto $1,2,3$, etc., contando a partir de $t=0$ do início da corrida) em que foi realizada a leitura (e.g., a da intensidade de $\mathrm{AM}(\mathrm{i}), \mathrm{CD}(\mathrm{n})$, etc.). O valor da intensidade $\mathrm{CD}(0)$ é calculado, evidentemente, utilizando o teor conhecido do elemento analisado na amostra CD, e os coeficientes $a$ e $b$ oferecidos pelo programa na curva de calibração desse elemento (ver equação linear de calibração, acima).

\section{Correçōes de background, interferên- cias e limites de deteç̧ão}

Correções de background são feitas apenas em elementos que apresentam diferenças significativas de background em matrizes diferentes; além de empregada para vários traços, considerou-se que também para o $\mathrm{Mg}$ elas seriam pertinentes (cf. Tabelas 2 e 3), particularmente quando se trabalha com ro- 
chas pobres nesse elemento.

Avaliações sobre interferências foram feitas em soluçōes sintéticas uni e multielementares. Não existem interferências sobre os elementos maiores, exceto uma superposição parcial de picos de $\mathrm{Mg}$ e Ca sobre a linha $766,491 \mathrm{~nm}$ do $\mathrm{K}$, que pode provocar erros significativos na análise de potássio em rochas ultramáficas se não corrigida. A Tabela 4 mostra as principais interferências observadas, calculadas a partir de soluções $1000 \mathrm{mg} / \mathrm{L}$ de elementos maiores (500 $\mathrm{mg} / \mathrm{L}$ de $\mathrm{Ti}$ ). Fe e $\mathrm{Ti}$ são os interferentes mais comuns; os outros elementos maiores não causam interferências significativas sobre as linhas utilizadas para análise de elementos traços.

Cálculos de limites de detecção foram feitos a partir de brancos da fusão alcalina e de materiais de referência também preparados por fusão. Os limites de detecção segundo os critérios da IUPAC (1978) são apresentados nas Tabelas 2 e 3 e correspondem à concentração equivalente a $3 \sigma$, onde $\sigma$ é o desviopadrão de 11 leituras do branco. A experiência mostra que os limites de detecção sob condiçōes de rotina são algo mais elevados, de modo que na prática adota-se limites de detecção equivalentes a $6 \sigma$, aqui chamados de $\mathrm{L}_{\mathrm{d}}$ (Tabela $3)$, enquanto $o$ valor de $3 \sigma$ é denominado limite de decisão $\mathrm{L}_{\mathrm{c}}$ (Massart et al., 1988). Quando presentes em teores inferiores a $\mathrm{L}_{d}$, os elementos traços são reportados como $\left(<\mathrm{L}_{d}\right)$. Também nas Tabelas 2 e 3 são indicados os limites de quantificação $\left(L_{c} ; 10 \sigma\right)$; teores entre $\mathrm{L}_{d}$ e $\mathrm{L}_{q}$ (Massart et al., 1988) se situam na "região de deteç̧ão" (ACS Committee on Environmental Improvement, 1980), mas carregam erro elevado, até acima de $50 \%$. Os valores de $\mathrm{L}_{\mathrm{q}}$ obtidos confirmam que a maior parte dos elementos maiores pode ser determinada quantitativamente quando em teores superiores a $0,1 \%$. Exceção notável é o $\mathrm{K}$, de dificil determinação em espectrome- tria de plasma; contudo, os resultados obtidos para rochas granitóides, com teores de $\mathrm{K}_{2} \mathrm{O}$ acima de $1 \%$, são plenamente satisfatórios. A maioria dos elementos traços tem $\mathrm{L}_{d}$ em torno de 10-15 ppm; para elementos como $\mathrm{Ba}, \mathrm{Sr}$ e $\mathrm{Zr}$, frequientemente presentes em teores superiores a $100 \mathrm{ppm}$, a determinaçăo em soluções preparadas por fusão alcalina é muito boa. Em certos casos, porém, os teores de elementos-traços em rocha estarão entre $\mathrm{L}_{d}$ e $\mathrm{L}_{q}$, ou mesmo abaixo de $\mathrm{L}_{\mathrm{d}}$ se resultados quantitativos são requeridos, é necessário considerável trabalho adicional, empregando outra metodologia de abertura (ataque ácido), que retira o Si da solução e usa menor diluição (1:100).

\section{RESULTADOS}

As Tabelas 5, 6 e 7 mostram resultados de análises efetuadas em materiais de referência utilizando o procedimento descrito acima.

$\mathrm{Na}$ Tabela 5 são apresentados os resultados de análises dos sete elementos maiores realizadas em cinco materiais de referência do Serviço Geológico do Japão (andesitos JA-1 e JA-2, granitos JG-3 e JG-1a e basalto JB-1a) e em um do Serviço Geológico da África do Sul (norito NIM-N), cobrindo o intervalo composicional basalto-granito ( 52 $72 \% \mathrm{SiO}_{2}$ ). A comparação entre os resultados recomendados (Govindaraju, 1994) e os obtidos mostra que a exatidão alcançada é plenamente satisfatória para todos os elementos maiores.

$O$ resultado apresentado para cada amostra corresponde à média de 3 ou 4 determinações realizadas em uma mesma corrida para uma mesma preparação. $O$ desvio-padrâo relativo $\left(\mathrm{RSD}_{\mathrm{T}}\right)$ observado é assim uma medida da repetibilidade e portando da eficácia do procedimento de correção de drifi adotado. Com uma exceção (amostra JA-2), a correção mantém os desvios-padrões re- 
Tabela 7 - Resultados das análises de precisão para longo intervalo de tempo (ca. 12 meses) em material de referência (JG-1a) e material de referência certificado (GS-N).

$\begin{array}{lrrrrr}\text { Elemento } & \begin{array}{r}\text { Valor } \\ \text { Recom. }\end{array} & \begin{array}{c}\text { GS-N } \\ \text { Valor }\end{array} & \begin{array}{r}\text { Obtido } \\ \mathrm{s}_{\mathrm{r}}\end{array} & \mathrm{RSD}_{\mathrm{r}} & \begin{array}{c}\mathrm{n}^{\circ} \text { de } \\ \text { obs. }\end{array} \\ \mathrm{SiO}_{2} & 65.80 & 65.85 & 0.65 & 0.98 & 13 \\ \mathrm{Al}_{2} \mathrm{O}_{3} & 14.67 & 14.51 & 0.21 & 1.45 & 13 \\ \mathrm{Fe}_{2} \mathrm{O}_{3} & 3.75 & 3.73 & 0.06 & 1.68 & 13 \\ \mathrm{MgO} & 2.30 & 2.31 & 0.04 & 1.59 & 13 \\ \mathrm{CaO} & 2.50 & 2.48 & 0.07 & 2.80 & 13 \\ \mathrm{Na}_{2} \mathrm{O} & 3.77 & 3.74 & 0.10 & 2.69 & 13 \\ \mathrm{~K}_{2} \mathrm{O} & 4.63 & 4.59 & 0.11 & 2.34 & 13 \\ \mathrm{TiO}_{2} & 0.68 & 0.67 & 0.02 & 2.48 & 13 \\ \mathrm{P}_{2} \mathrm{O}_{5} & 0.28 & 0.29 & 0.01 & 4.98 & 13 \\ \mathrm{MnO} & 0.056 & 0.056 & 0.004 & 7.30 & 13 \\ & & & & & \\ \mathrm{Ba} & 1400 & 1380 & 26 & 1.89 & 12 \\ \mathrm{Sr} & 570 & 571 & 10 & 1.70 & 12 \\ \mathrm{Zr} & 235 & 227 & 15 & 6.60 & 6 \\ \mathrm{Y} & 16 & 16 & 1 & 4.39 & 3 \\ \mathrm{La} & 75 & 73 & 13 & 17.25 & 7 \\ \mathrm{~V} & 65 & 78 & 4 & 4.78 & 11\end{array}$

\begin{tabular}{|c|c|c|c|c|c|}
\hline & & JG-1a & & & \\
\hline Elemento & $\begin{array}{l}\text { Valor } \\
\text { Recom. }\end{array}$ & $\begin{array}{l}\text { Valor } \\
\text { Obtido }\end{array}$ & $s_{T}$ & $\mathrm{RSD}_{\mathrm{r}}$ & $\begin{array}{l}\mathrm{n}^{e} \mathrm{de} \\
\text { obs. }\end{array}$ \\
\hline $\mathrm{SiO}_{2}$ & 72.19 & 72.34 & 0.90 & 1.24 & 8 \\
\hline $\mathrm{l}_{2} \mathrm{O}_{3}$ & 14.22 & 14.05 & 0.14 & 1.03 & 9 \\
\hline $\mathrm{Fe}_{2} \mathrm{O}_{3}$ & 2.05 & 2.05 & 0.02 & 1.11 & 9 \\
\hline $\mathrm{MgO}^{\prime}$ & 0.69 & 0.72 & 0.04 & 5.22 & 9 \\
\hline $\mathrm{CaO}$ & 2.13 & 2.17 & 0.04 & 1.98 & 9 \\
\hline $\mathrm{Na}_{2} \mathrm{O}$ & 3.41 & 3.39 & 0.05 & 1.57 & \\
\hline $\mathrm{K}_{2} \mathrm{O}$ & 4.01 & 3.96 & 0.06 & 1.63 & 9 \\
\hline $\mathrm{TiO}_{2}$ & 0.25 & 0.26 & 0.01 & 4.64 & 9 \\
\hline $\mathrm{P}_{2} \mathrm{O}_{5}$ & 0.08 & 0.09 & 0.02 & 17.82 & 9 \\
\hline Inó & 0.06 & 0.062 & 0.004 & 5.70 & 9 \\
\hline & 458 & 467. & 9 & 1.89 & \\
\hline & 185 & 184 & 2 & 1.19 & 9 \\
\hline Ir & 121 & 122 & 19 & 15.69 & \\
\hline & 31.6 & 30 & 2 & 6.22 & \\
\hline $\mathrm{La}$ & 21.8 & 25 & 9 & 35.38 & 8 \\
\hline & 23 & 14 & 3 & 20.64 & 3 \\
\hline
\end{tabular}

lativos abaixo ou em torno de $1 \%$ para os óxidos presentes em maior concentração. O desempenho menos satisfatório é observado para os álcalis, que reconhecidamente oferecem maiores di- ficuldades em espectrometria de plasma, já que produzem sinal mais instável em intervalos de tempo curto (como mostrado pelos maiores $\mathrm{RSD}_{\mathrm{r}}$ nas leituras individuais, tipicamente superiores a $1 \%$ ) e também nos de longo intervalo (drift mais acentuado; e.g., Walsh, 1992). No caso do potássio, adicionalmente, a única linha utilizável $(766,491$ $\mathrm{nm})$ tem sensibilidade muito baixa.

Os valores mais altos do desviopadrão relativo observados na amostra JA-2 (entre 1,0 e 2,8\%) refletem principalmente a influência de variações de curto intervalo, que não podem ser corrigidas sem o emprego de padrões internos, procedimento inviável em equipamentos com espectrômetro sequiencial. O principal efeito dessas variaçōes é uma flutuação geral, para cima ou para baixo, de todo o conjunto de elementos analisados, de modo que as proporções entre eles são mantidas, embora em função disto o fechamento tipicamente observado nas análises varie entre 98 e $101 \%$.

Os resultados acima mostram que o procedimento de correção empregado melhora sensivelmente o desempenho do equipamento, viabilizando a obtenção de análises de elementos maiores com qualidade plenamente satisfatória para uso em problemas petrológicos. O controle de qualidade efetuado com a inserção de materiais de referência certificados em todas as corridas garante a identificação de eventuais casos em que a correção não foi efetiva para minimizar os efeitos do dirift (por exemplo, quando o drift é acentuado e fortemente não-linear em um intervalo entre duas amostras de controle), quando então as amostras devem ser reanalisadas.

A Tabela 6 mostra resultados obtidos para elementos menores e traços para quatro materiais de referência (granitos GH e GS-N, granodiorito JGla e basalto JB-la), em rotina análoga à descrita acima. A correção de drift 
resulta em sensivel ganho de repetibilidade para todos os elementos citados, embora naturalmente exatidāo e repetibilidade sejam deteriorados nos casos em que os teores observados se encontram abaixo de $\mathrm{L}_{q}$ (caso típico sendo o La, cujos teores na maioria dos casos encontram-se entre $L_{c} e L_{d}$ ). Dentro do conjunto analisado, destacam-se os excelentes resultados obtidos para alguns elementos que estão tipicamente presentes em teores muito acima de $\mathrm{L}_{q}$ e que emitem linhas de elevada sensibilidade no equipamento $(\mathrm{Ba}, \mathrm{Sr}$ e $\mathrm{MnO}$, este último com exatidão na terceira casa decimal). Em contrapartida, $\mathrm{P}_{2} \mathrm{O}_{5}$ acompanha o $\mathrm{La}$ como analito mais dificil do conjunto, em particular devido à baixa sensibilidade e $\mathrm{RSD}_{\mathrm{I}}$ relativamente elevado (até 2-3\%) da linha utilizada $(213,618 \mathrm{~nm})$ e à presença de várias linhas de $\mathrm{Fe}$ próximas, dificultando a correçăo de background, estritamente necessária neste caso devido à radiação de fundo provocada pelo Al nessa regiāo. A opção pela linha $178,29 \mathrm{~nm}$ do $\mathrm{P}$, na região do ultravioleta, adotada por alguns autores, mostra-se menos favorável, em especial devido ao elevado RSDr das leituras individuais (até $3-4 \%$ ). A despeito das dificuldades apontadas, os resultados obtidos são excelentes para teores de $\mathrm{P}_{2} \mathrm{O}_{5}$ acima de $0,05 \%$, o que inclui a quase totalidade das amostras analisadas no Laboratório.

A Tabela 7 apresenta os valores médios de concentrações determinadas ao longo de cerca de 12 meses em diferentes preparaçóes dos dois materiais de referência (granodiorito JG-1a e granito GS-N), utilizados como controle de qualidade nas análises de rochas graniticas realizadas no Laboratório. Variaçōes do desvio padrão relativo em torno de $1 \%$ para os óxidos considerados críticos $\left(\mathrm{SiO}_{2}, \mathrm{Al}_{2} \mathrm{O}_{3}, \mathrm{Fe}_{2} \mathrm{O}_{3}\right.$ ) atestam a boa precisão alcançada na fase de implantação da metodologia. Novamente, alguns valores relativamente elevados devemse exclusivamente aos baixos teores do analito (casos do $\mathrm{MgO}$ e $\mathrm{P}_{2} \mathrm{O}_{5}$ na amostra JG-la e MnO na amostra GS-N). A aplicação de método estatístico (teste t) para verificação da concordância entre os valores obtidos e aqueles certificados só foi possível para o material de referência GS-N, visto o granito JG-1a possuir apenas valores recomendados. Os resultados da aplicação deste teste sobre os dados apresentados na Tabela 7 indicam que, para uma confiabilidade de $95 \%(\alpha=0.05)$, apenas o vanádio apresenta desvio (bias) positivo real para o número de dados amostrados.

$\mathrm{O}$ número menor de determinaçōes obtidas para $\mathrm{Zr}$, La e Y reflete o fato de que as análises iniciais obtidas no laboratório não incluíam esses elementos, que ainda apresentavam problemas. A determinação precisa do $\mathrm{Zr}$, cuja importância em petrogênese de rochas graníticas tem sido mais e mais destacada, só foi possível após a introdução de modificações no procedimento de preparação de amostras adotado originalmente. Apenas com a adição de HF (2 gotas $/ 50 \mathrm{~mL}$ ) à soluçăo foi possivel obter resultados quantitativos; sem isto, parte do $\mathrm{Zr}$ não se mantém dissolvido mesmo nas primeiras horas após a solubilizaçāo.

Ao longo do primeiro ano de desenvolvimento de rotina analítica, o Laboratório de ICP-AES do DMP-IG, da USP, produziu cerca de duas centenas de determinações de elementos maiores, menores e traços a partir de soluções obtidas por fusão alcalina, principalmente de rochas graniticas, com resultados perfeitamente consistentes com a petrografia previamente disponivel (e.g., Janasi et al., 1995; Janasi, 1995). No momento, desenvolvem-se rotinas para análise de elementos-traços a partir de aberturas por ataque ácido (que permitem em certos casos melhorar os limites de deteç̧ẫo em até uma ordem de gran- 
deza) e para elementos terras-raras a partir da combinação de abertura por fusão alcalina e separação em colunas de troca iônica.

\section{AGRADECIMENTOS}

Os autores agradecem o apoio da FINEP na aquisição do espectrômetro de emissâo atômica com plasma induzido ARL-3410 e na infra-estrutura laboratorial. À FAPESP (Processos 93/4442 -2 e 93/3623-3) e ao CNPq (através de Taxas de Bancada) os agradecimentos pelo apoio à compra de materiais de consumo e aqueles de manutenção rotineira do espectrômetro e do laboratório.

\section{REFERÊNCIAS BIBLIOGRÁFICAS}

\section{ACS COMITTEE ON ENVIRON-} MENTAL IMPROVEMENT (1980) Guidelines for data acquisition and data quality evaluation in environmental chemistry. Analytical Chemistry, v.52, p.2242-2249.

CANTAGREL, F.; PIN, C. (1994) Major, minor and rare-earth element determinations in 25 rock standards by ICP-Atomic Emission Spectrometry. Geostandards Newsletter, v.18, n. 1, p.123-138.

CARRE, M.; POUSSEL, E; MERMET, J.M. (1992) Drift diagnostics in Inductively Coupled Plasma Atomic Emission Spectrometry. Journal of Analytical Atomic Spectrometry, v.7, p.791-797.

DUTRA, C.V. (1984) Método para determinação de traços e subtraços de terras raras em rochas por espectrometria de plasma (ICP) - aplicação em petrogênese. In: CONGRESSO BRASILEIRO DE GEOLOGLA, 33., Rio de Janeiro, 1984. Anais. Rio de Janeiro, SBG, v.10, p.4792-4805.

FIGUEIREDO, M.C.H. (1985) Introdução à geoquímica dos elementos terras raras. Boletim IG-USP. Série
Cientifica, v.16, p.15-31.

GLADNEY, E.J.; JONES, E.A.; NICKELL, E.J; ROELANDTS I. (1990) 1988 compilation of elemental concentration data for USGS Basalt BCR-1. Geostandards Newsletter, v.14, n.2, p.209-359.

GOVINDARAJU, K. (1994) 1994 compilation of working values and sample description for 383 geostandards. Geostandards Newsletter, v.18 (Special Issue), p.1-158.

GOVINDARAJU, K. (1995) 1995 working values with confidence limits for twenty-six CRPG, ANRT and IWG-GIT geostandards. Geostandards Newsletter, v.19 (Special Issue), p.1-32.

IUPAC - International Union of Pure and Applied Chemistry (1978) Nomenclature, symbols, units and their usage in spectrochemical analysis III. Analytical flame spectroscopy and associated non-flame procedures. Spectrochimica Acta, v.33B, p.247269.

IUPAC - International Union of Pure and Applied Chemistry (1990) Harmonized protocols for the adoption of standardized analytical methods and for the presentation of their perfomance characteristics. Pure \& Applied Chemistry, v. 62 , n.1, p.149-162.

IUPAC - International Union of Pure and Applied Chemistry (1993) The international harmonized protocol for the testing of (chemical) analytical laboratories. Pure \& Applied Chemistry, v.65, n.9, p.2123-2144.

JANASI, V.A. (1995) Geochemical constraints on the mangerite-"rapakivi" granite connection in the Neoproterozoic São José do Rio Pardo suite, southeastem Brazil. In: SYMPOSIUM ON RAPAKIVI GRANITES AND RELATED ROCKS, Belém, 1995. Abstracts Volume, p.42-43. 
JANASI, V.A.; CUCHIERATO, G.; ULBRICH, H.H.G.J. (1995) Geoquímica de elementos maiores e traços em granitos maciços Pilar do Sul e Serra Bateia, SP: resultados de análises por ICP-AES. In: SIMPÓSIO DE GEOLOGIA DO SUDESTE, 4., Águas de Sâo Pedro, SP, 1995. Boletim de Resumos, p.133.

JARVIS, I; JARVIS, K.E. (1992) Plasma spectrometry in the earth sciences: techniques, applications and future trends. Chemical Geology, v. 95, p. 1-33.

MASSART, D.L.; VANDEGINSTE, B.G.M.; DEMING, S.N.; MICHOTTE, Y; KAUFMAN, L. (1988) Chemometrics: a textbook. New York, USA, 488p.

POTTS, P.J. (1987) A Handbook of Silicate Rock Analysis. Blackie, Glasgow-Londres, 622p.

POTTS, P.J.; KANE, J.S (1992) Terminology for geological reference material values: a proposal to the International Organisation for Stan- dardisation (ISO), producers and users. Geostandards Newsletter, v. 16, n.2, p.333-341

SULCEK Z; POVONDRA P. (1989) Methods of Decomposition in Inorganic Analysis. CRC Press, Boca Raton, 325p.

THOMPSON, M.; WALSH, J.N. (1989) Handbook of Inductively Coupled Plasma Spectrometry. 2.ed., Blackie, Glasgow-Londres, $316 \mathrm{p}$.

WALSH, J.N. (1992) Use of multiple internal standards for high-precision, routine analysis of geological samples by inductively coupled plasmaatomic emission spectrometry. Chemical Geology, v.95, p.113-121.

WALSH, J.N.; HOWIE, R.A. (1980) An evaluation of the performance of an inductively coupled plasma source spectrometer for the determination of the major and trace constituentes of silicate rocks and minerals. Mineralogical Magazine, v.43, p.967-974.

V.A.Janasi - Instituto de Geociências, Universidade de São Paulo, Caixa Postal 11.348, CEP 05422-970, São Paulo, SP, Brasil. 